\title{
CSR and leadership: can China lead a new paradigm shift?
}

\author{
Peter Buyaert
}

Published online: 13 July 2011

(C) Springer Science+Business Media B.V. 2011

\begin{abstract}
Globally, corporate social responsibility (CSR) needs to find its sustainable development via the recognition of tangible benefits that CSR will bring to organizations and their stakeholders. The less tangible but likely most important benefit lies in the continual improved leadership and management quality emerging from organizations investing in CSR. Companies' failure to act in a CSR way and the lack of wise leadership and quality management is a dominant root factor in the past scandals and financial crisis. Looking at current interactions between Chinese companies and their various influencing environmental, society and government (ESG) factors, many of the motivational drivers for Western companies to invest in CSR are today still being challenged here based on China's market specifics. More rapid development of the regulatory framework with adequate enforcement resources, as well as incorporation of CSR "value" education at universities can accelerate this needed development. In the short term, foreign companies as "guests" of China should also be a catalyst as role models. Apart from a foreign investor's actions, it is equally important to acknowledge and address local China challenges remaining to provide the right climate for a foreign company's CSR programs to be successfully executed here. The world is at a cross roads of a new value-based leadership paradigm. With global interdependence accelerating and hence, management and leadership complexity expanding, there is a need for a new leadership model, bringing "common wisdom" more pronounced to the forefront, arising from a renewed focus and strengthening of an aligned set of values between family, society, and corporate life. China's unique demographic challenges and economic progress provide a fertile environment to lead the world in this development.
\end{abstract}

Keywords CSR · Leadership · Wisdom · CSR drivers · China challenges · Regulatory development $\cdot$ Paradigm shift $\cdot$ Society values

P. Buyaert $(\bowtie)$

China Global Leaders, Pudong, China

e-mail: peter.buytaert@ChinaGlobalLeaders.com 


\section{Introduction}

Corporate social responsibility (CSR) definitions vary widely albeit mostly centering round the same themes of ethical, environmental correctness, and legal compliance by business enterprises, e.g., the version endorsed by the European Commission http://ec.europa.eu/ enterprise/policies/sustainable-business/corporate-social-responsibility/index_en.htm is:

"A concept whereby companies decide voluntarily to contribute to a better society and a cleaner environment. A concept whereby companies integrate social and environmental concerns in their business operations and in their interaction with their stakeholders on a voluntary basis."

We subscribe to a model that relates a company's culture, governance structure, and actions to the "ESG" factors (environment, society and government) it operates in. We assess corporate social responsibility by looking at an organization's ethical and socially responsible processes and decisions across all three factors and relate them to what motivates all internal and external stakeholders to participate and/or endorse. Though "moral" principles should generally guide individuals in their decision making, too often an individual's morality can be compromised by the "greater goal" of stakeholders, organizations or society with differing objectives and latent opportunities.

This approach provides a useful framework to explore and identify the motivation and drivers for organizations to ultimately and sustainably invest in corporate social responsibility, rather than engaging in one time public relation programs.

In our view, CSR can only excel and be sustained via the alignment between all stakeholders (government, non-government organizations, consumers, etc.) promoting, incentivizing, and ultimately rewarding CSR and companies delivering value in return via their CSR programs.

If investments in CSR programs prove to return bottomline and brand value, alignment of a company's stakeholders around these programs is fairly easy and a CSR "culture" will ultimately be embedded in the organization. Without the correct motivational factors, for any organization to invest in CSR is highly unlikely and not sustainable.

Based on the various interactions of an organization with its employees, regulatory authorities, customers, and other stakeholders, CSR actions can return value in various ways:

- The sustainability of the organization via regulatory and legal compliance, as well as a professional risk management approach;

- The improved quality of leadership and reduced employee turnover, rallying an organization's resources around a set of ethically correct and socially responsible values linking employees to an "admirable" company, rather than a purely financially rewarding job. This in itself will result in potentially substantial HR cost reductions and better management decisions

- Competitive differentiation appealing to a growing consumer market with CSR awareness

- Taking a lead in regulatory compliance, hence pushing competitors to follow suit

- Increased brand value via an organizations' public CSR actions, directly increasing equity valuations 


\section{CSR and China}

When we look at the interactions between Chinese companies and their various influencing ESG factors, we observe that many of the motivational drivers for Western companies to invest in CSR are today still being challenged here based on China market specifics:

- Where in Europe and North America sustainability is a focus in a developed market with rather limited growth rates, China's market offers exceptional growth opportunities. Under these circumstances, successful management of this rapid expansion has priority over sustainability actions

- Directly linked to these growth dynamics, Chinese stakeholders' request for companies to also deliver on these growth expectations does not encourage investments in compliance and socially responsible actions

- With a regulatory framework generally still evolving, focus of organizations tends to be on execution with compliance running in the distance or simply nonapplicable due to inadequate regulations or lack of enforcement resources

- An exceptionally successful Chinese growth model tends to stimulate risk taking more than risk management at this point of its exponential growth

- With regards to competitive differentiation, only recently Chinese "brands" are emerging, potentially gaining from a CSR brand equity approach. To-date, most successful international Chinese players achieved their growth and stakeholder value via an original equipment manufacturer (OEM) supply approach to established global brands

- Last but not least, the emergence of the public markets and increased initial public offering activity should benefit CSR long term. However, to-date, private entrepreneurs are naturally motivated to prioritize "cash" over longer*term capital value returns via CSR actions

We believe though that despite these current observations, one can be optimistic about the natural evolving of interest by Chinese companies in CSR as each of the aforementioned factors will gradually develop. More rapid development of the regulatory framework with adequate enforcement resources, as well as incorporation of CSR "value" education at universities can accelerate this needed development.

In the short term, foreign companies as "guests" of China should also be a catalyst as role models. They should recognize the immediate benefits CSR has in competitive differentiation. Their actions to raise the bar in compliance and social responsibility should further be applied as benchmarks by local regulatory authorities. This will eventually push domestic competition to follow suit.

Foreign investors gain immediate benefits associated with this approach:

- Codes of conduct to be signed by all employees, explained and "lived", creating a transparent management tool

- Ethical behavior as a self-strengthening process via increased quality of labor force

- Value-based companies are admired by the new generation labor force

- Better retention correlates directly to reduced costs and stronger performance 
In several cases though, we also see foreign-invested companies not fundamentally addressing core issues of CSR and "walking the talk" but instead applying "make up", using CSR as a public relations tool.

In order to address CSR fundamentally and have it embedded in the organization, some of the basic questions in this respect to be addressed are:

- When outsourcing for lower wages in China (and other outsourcing markets), how large a wage differential is acceptable? Also, how to define the level for acceptability?

- Should a company accept health and safety standards in plants or facilities in China below those of its home country, or allow longer working hours, less overtime, or generally worse working conditions for staff than in its home country?

- Does a company provide audits to the external OEM providers on its environment and compliance policies?

- Should a buyer company demand that a supplier observes applicable national and local law and how many organizations have this consistently and explicitly addressed in their purchase agreements?

While there is undoubtedly room for improvement on these issues, it is equally important to acknowledge and address local China challenges remaining to provide the right climate for a foreign company's CSR programs to be successfully executed here. Some observations:

- The need for an independent and secure whistleblower channel in case of 'demand driven' corruption practices (not "pushed" or "demanded" by the foreign party)

- Effective execution and enforcement of laws and regulations at a level playing field for both foreign and domestic investment

- Given the well-known challenge of "numbers" in China, a potential focus and priority to be allocated to "life threatening" non-compliance areas (e.g., cases of pharmaceuticals and food related non-ethical behaviors and regulatory violations), rather than trying to address all areas

\section{Towards a new leadership paradigm}

There is undoubtedly progress being made on corporate social responsibility in China, nevertheless fundamental challenges remain. These challenges are directly linked to the specific market dynamics. Education of "value-based leadership", as well as strengthened regulatory compliance and enforcement will both accelerate successful development of CSR here.

Globally, CSR needs to find its sustainable development via the recognition of tangible benefits that CSR will bring to organizations and their stakeholders. The less tangible but likely most important benefit lies to our opinion in the continual improved leadership and management quality emerging from organizations investing in CSR. We argue that wise leadership and better management quality will automatically lead to an organization's cost optimizations, increased brand value, 
and sustainable growth via aligned and continued ethically correct and socially responsible decisions and actions.

The lack of wise leadership and quality management in a world growing more integrated, yet more virtually complex to manage, is to our view a dominant root factor in the past scandals and financial crisis, that we are still trying to come to grips with today.

China also here has a unique opportunity to leapfrog and accelerate leadership in CSR from these lessons learned.

In our view, the world is at a cross roads of a new value-based leadership paradigm. With global interdependence accelerating and hence, management and leadership complexity expanding, there is a need for a new leadership model, bringing "common wisdom" more pronounced to the forefront, arising from a renewed focus and strengthening of an aligned set of values between family, society, and corporate life. This alignment can only be achieved via an increased investment in the appropriate value education, a transparent and adjusted legal and regulatory framework, addressing societal changes, as well as the insurance that basic needs of all citizens are being addressed. The latter also seeing an increasing dependence on a redistribution of private wealth, rather than via public organizations.

This new model can undoubtedly also provide rise to a new breed of socially responsible entrepreneurs, as well as social enterprises, applying a corporate model to address increasing societal challenges today, but motivated by socially responsible actions.

China's unique demographic challenges and economic progress provide a fertile environment to lead the world in this development. 